\title{
ARCHITECTURE OF HTS LEADS SOFTWARE PROTECTION SYSTEM
}

\author{
J.M.Nogiec" ${ }^{\#}$, S.Feher, D.F.Orris, J.Sim, M.Tartaglia, \\ Fermi National Accelerator Laboratory ${ }^{*}$, Batavia, Illinois 60510
}

\begin{abstract}
A software system to detect quenches in high temperature superconducting (HTS) power leads has been developed as a part of the Fermilab HTS R\&D program. The system has been successfully applied to protect leads during several tests of various types of HTS leads.

The software architecture of the configurable quench detection system and capabilities of the quench management system are described. All major system components are presented along with the solutions to their communication and distribution problems. In addition, failure protection aspects of the design are discussed. Graphical user interfaces used to monitor the quench protection system are also presented.
\end{abstract}

\section{INTRODUCTION}

High temperature superconducting leads have been considered at Fermilab as a cost-effective replacement of traditional power leads. Thus, a new system has been built to test and study performance of these leads. This system is based on the Distributed Monitoring and Control System (DMCS) developed at Fermilab.

The DMCS [1][2] provides a framework for constructing data acquisition, monitoring and control systems, and has been previously successfully applied to control cold tests of superconducting accelerator magnets [3], and studies of future accelerator magnets (Pipetron project [4]).

The DMCS is a decentralised, configurable, multiplatform system with open architecture. Core components of this system include (see Figure 1):

- A memory resident Real-Time Data Base (RTDB) supporting triggers, caching, and locking mechanisms, and providing transparent access to local and remote objects

- A scan system that supports a uniform, common approach to data archiving, data acquisition, control, and on-line processing through various scan types

- A distributed synchronisation and communication mechanism based on a software bus approach, that enables synchronisation between active system components such as scans and servers

"Operated by the Universities Research Association under contract with the U.S. Department of Energy

"Email: nogiec@fnal.gov
- A configuration subsystem with Data Base Definition Language describing system configurations

- Unified user interfaces that enable monitoring of the system under control (trends, numerical values, overviews), and status of the communication and scan subsystems

- Off-line data analysis tools.

The state of the process under control is monitored and altered through manipulations done on a set of process variables (PVs). Some PVs reflect directly measured process parameters, whereas others contain control variables used to regulate the process. Still other PVs may contain system control parameters, internal system variables, statistics, long-term trends, etc.

The scan system links the RTDB with the process under control by uniformly accessing devices and pseudodevices and passing data to and from the RTDB. In addition to the already mentioned PVs, RTDB also contains all system configuration data.

The core system components are supplemented by specialised subsystems, such as the power supply control system [5], to form the full-featured operating environment.

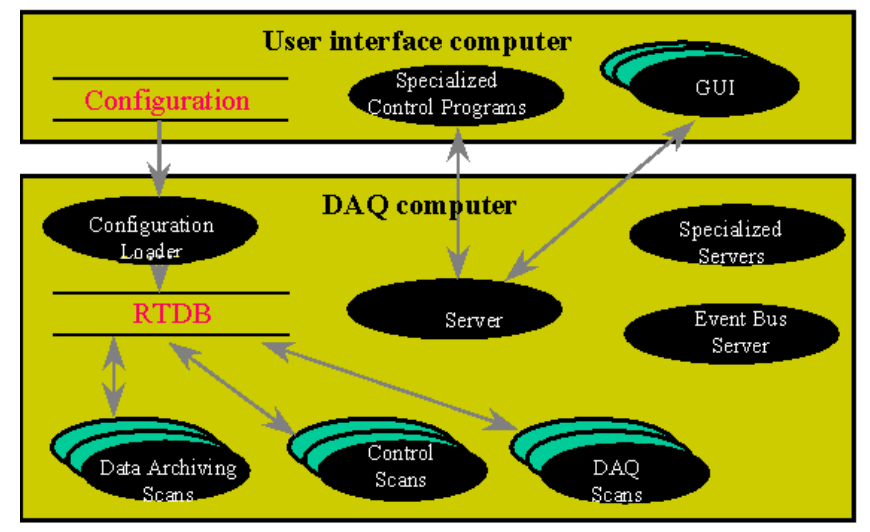

Figure 1: Overview of DMCS

\section{REQUIREMENTS}

The moderate quench development speeds in high temperature superconductors, measured in seconds, allow for software-based quench protection systems. Despite the fact that the system does not have hard real-time requirements, it still has to be deterministic. The protection system should also be highly reliable to 
adequately protect the HTS leads. These requirements were crucial to the construction of a new software quench protection system.

\section{ARCHITECTURE OF QUENCH PROTECTION SYSTEM}

The quench protection system consists of a set of components distributed in a multi-platform computer system. Functionally, the system follows a traditional, hierarchical approach with user interfaces running on workstations and direct control and data acquisition parts running on VME-based computers under control of realtime operating systems.

\subsection{Quench System}

The quench system comprises the quench detection, quench management, and quench characterisation subsystems.

The software HTS quench detection subsystem relies on both voltage taps and RTD temperature sensors for the determination of a quench. These voltages and temperatures are monitored continuously by independent asynchronous scans. The triggering mechanism of the RTDB is used to signal when monitored values exceed pre-set alarm limits. The scans communicate with the software quench manager which monitors status of the software quench detection system and informs the central quench manager about detected quenches or malfunctions. The software quench manager also initiates the power supply ramp-down sequence in order to protect the HTS leads. The central quench manager communicates with the software quench detection subsystem and the hardware-based backup quench detection system [6]. It also signals quench characterisation scans to trigger data loggers that record quench signals and subsequently store quench data and latch change of state (COS) information.

The quench system components, as described above, are located on several computers (Figure 2) and communicate exclusively through events.

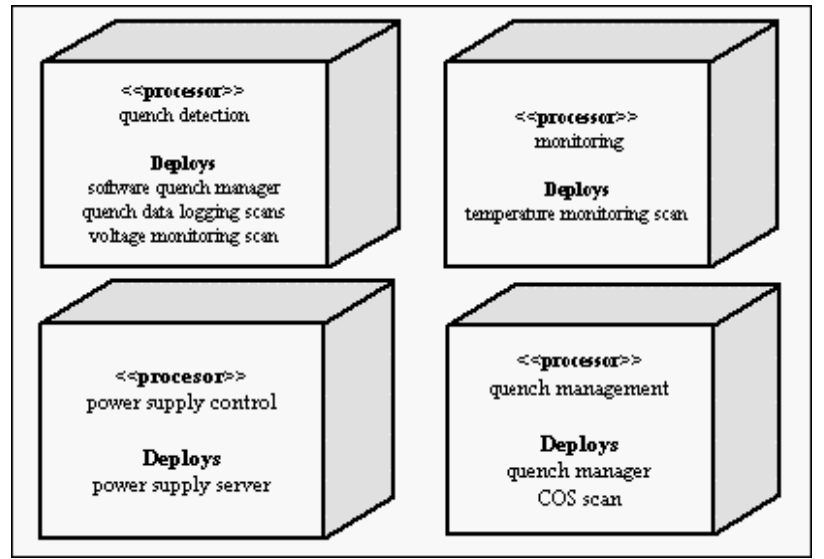

Figure 2: Deployment diagram

\subsection{User Interfaces}

Several graphical user interfaces are available to monitor and control the quench system:

- An event monitor capable of reporting occurrences of selected, in this case quench-related, events and capturing sequences of events (Figure 3)

- A scan control panel monitoring status of the scanning system and allowing scan attribute manipulation at run-time

- Process variable display interfaces showing instantaneous values of monitored voltages and temperatures, their trends and alarm statuses

- A quench manager panel displaying quench-related information and allowing manual control over the quench system

- A power supply control panel.

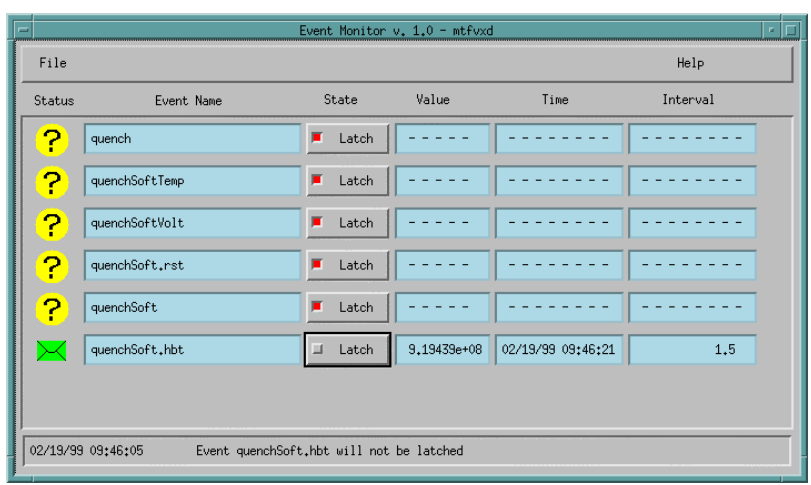

Figure 3: Event monitor

The overview of the HTS leads under test is shown in Figure 4.

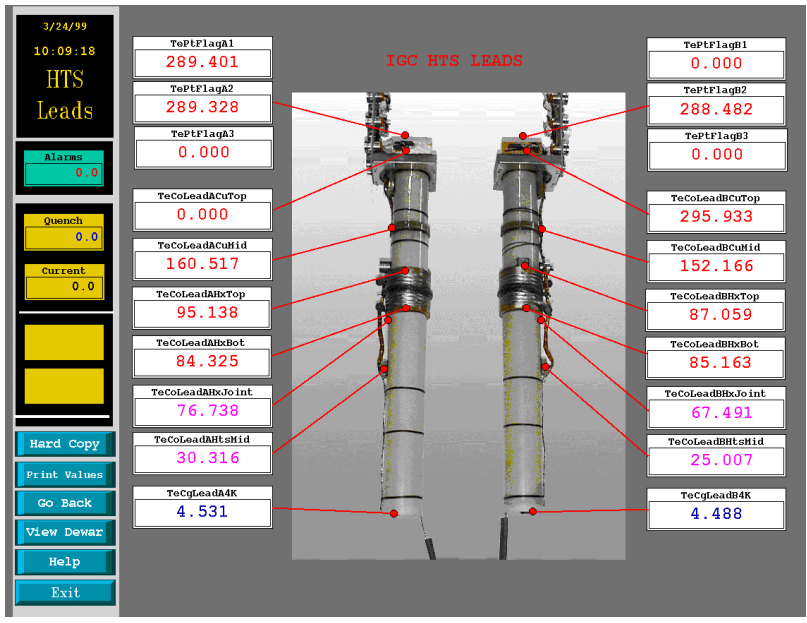

Figure 4: Overview of HTS leads 


\section{INTERACTIONS IN QUENCH PROTECTION SYSTEM}

Components of the software quench protection system interact by sending and receiving events. The interactions which take place upon detection of a quench are shown using a collaboration diagram (Figure 5).

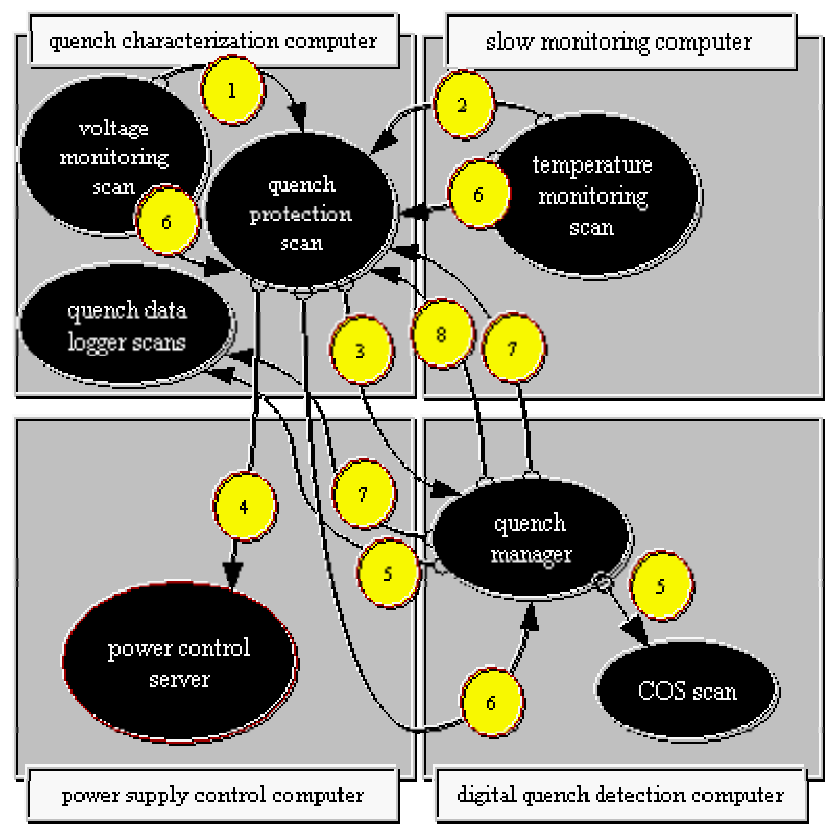

Figure 5: Quench system interaction diagram

Upon detection of a quench by one of the monitoring scans (temperature or voltage), an appropriate event is sent to the software quench manager $(1,2)$. The software quench manager informs the central quench manager about the quench detected by the software quench protection system (3) and sends a request to the power supply server to ramp the current down (4). Afterwards, fast data logging scans are triggered to latch and save quench data (5).

If a quench is detected first by the hardware detection system the software quench manager is informed about it by the quench manager (8).

Quench status is reset by the quench manager in response to the user request sent from the quench manager user interface (7) which restarts the whole quench system and allows for ramping current in the leads.

The components vital to quench protection periodically exchange heartbeat events to detect any malfunctions of the system (6).

\section{SYSTEM FEATURES}

The required high reliability of the system has been achieved through the distribution of system components, independent voltage and temperature monitoring, implementation of heartbeat signals, and usage of a backup hardware quench detection system.
Determinism of the quench protection system, important in the case of a software system, has been guaranteed by using a real-time operating system as a system run-time environment.

The software quench protection system can be easily reconfigured and adapted to accommodate various HTS leads. Among others, the number and location of sensors can be modified, new quench detection scans can be added, and thresholds used to determine presence of a quench in the leads can be modified.

\section{CONCLUSIONS}

The moderate quench development speeds in HTS leads make it possible to rely on software systems for quench protection. Software -based systems can offer potentially high flexibility and configurability in comparison with purely hardware-based systems.

The required high reliability can be achieved through duplication and distribution of system components and deployment of a backup hardware solution.

Relying on a common framework makes integration of the quench system with the rest of the monitoring and control system seamless.

Basing the implementation of the quench system on a configurable and open system makes this system extensible and allows for:

- modification of quench detection methods or algorithms;

- introduction of other quench protection mechanisms;

- modification of quench characterisation elements; and,

- modification of the interactions between system components.

\section{REFERENCES}

[1] J.M.Nogiec, E.Desavouret, D.Orris, J.Pachnik, S.Sharonov, J.Sim, J.C.Tompkins, and K.Trombly-Freytag, "A Distributed Monitoring and Control System", PAC'97, Vancouver, (1997)

[2] J.M.Nogiec, E.Desavouret, J.Pachnik, S.Sharonov, and J.Sim, "An Open Distributed Monitoring and Control System", CHEP'97, Berlin, (1997)

[3] M.J.Lamm, J.DiMarco, E.Desavouret, S.Feher, J.D.Garvey, C.Hess, P.J.Limon, J.M.Nogiec, D.F.Orris, J.Pachnik, T.Peterson, S.Sharonov, J.B.Strait, C.Sylvester, J.W.Sim, M.Tartaglia, J.C.Tompkins, and A.V.Zlobin, "A New Facility to Test Superconducting Accelerator Magnets", PAC'97, Vancouver, (1997)

[4] G.W.Foster, P.O.Mazur, T.Peterson, C.D.Sylvester, and P.Schlabach, "Design and Operation of an Experimental Double-C Transmission Line Magnet", PAC'97, Vancouver, (1997)

[5] S.Sharonov, J.M.Nogiec, "An Embedded Power Supply Controller", Pac'97, Vancouver, (1997)

[6] D.F. Orris, S. Feher, M.J. Lamm, J. Nogiec, S. Sharonov, M. Tartaglia, and J. Tompkins, "Digital Quench Detection System for Superconducting Magnets", PAC'99, New York, (1999) 